\title{
Population trapping due to cavity losses
}

\author{
M. Scala,, , 7 B. Militello, ${ }^{1}$ A. Messina,${ }^{1}$ S. Maniscalco,${ }^{2}$ J. Piilo, ${ }^{2}$ and K.-A. Suominen ${ }^{2}$ \\ ${ }^{1}$ MIUR and Dipartimento di Scienze Fisiche ed Astronomiche \\ dell'Università di Palermo, via Archirafi 36, I-90123 Palermo, Italy \\ ${ }^{2}$ Department of Physics, University of Turku, FI-20014 Turun yliopisto, Finland
}

\begin{abstract}
In population trapping the occupation of a decaying quantum level keeps a constant non-zero value. We show that an atom-cavity system interacting with an environment characterized by a non-flat spectrum, in the non-Markovian limit, exhibits such a behavior, effectively realizing the preservation of nonclassical states against dissipation. Our results allow to understand the role of cavity losses in hybrid solid state systems and pave the way to the proper description of leakage in the recently developed cavity quantum electrodynamic systems.

PACS numbers: 42.50.Pq, 42.50.Lc, 03.65.Yz
\end{abstract}

Population trapping arises when a multilevel system interacting with external driving fields is frozen in a given state for very long times [1, 2, 3]. The phenomenon was originally discovered in the dynamics of systems with at least three discrete levels [1]. The same phenomenon was shown to appear in systems with a continuum of levels [2] and in quantum systems interacting with quantized fields [3]. In all of these cases the mechanism is almost the same: population trapping occurs when there exists a superposition of states which decouples from the other states, so that its population is constant in time. In this case, the various transition channels corresponding to the states in the superposition interfere destructively, canceling the decay [2]. An analogous phenomenon has been singled out in the dissipative dynamics of an atom interacting with a structured reservoir. Indeed, for a twolevel atom interacting with the quantized e.m. modes of a photonic-bandgap (PBG) material [4, 5], the atomic population can be partially trapped in the excited state, when the atomic Bohr frequency is near the edge of the gap [6, 7, 8,6$]$. In this case the trapping is due to the formation of two atom-photon dressed states, one of which, due to strong vacuum Rabi splitting, is protected against decay because the energy of the relevant transition to the ground state lies inside the gap, as explained in Ref. [9]. This has led to an extensive theoretical and experimental analysis of the physics of cavities inside PBG materials interacting either with real atoms [10] or quantum $\operatorname{dots}[11,12]$.

As discussed in Ref. [9], what was missing until some years ago was the inclusion of cavity losses in the study of the dynamics of these systems. The cavity losses can be thought as due to an imperfect realization of the experimental setup for the study of these systems. More precisely they arise from the imperfect alignment of the auxiliary waveguides which couple to the cavities according to the designed configurations [11]. Recently the effect of cavity losses has been studied using a phenomenological dissipator [10, 11], identical to the one used to

*Electronic address: matteo.scala@fisica.unipa.it describe the losses in cavity quantum electrodynamics (CQED) 13. However, one may wonder if and in which limits this phenomenological model is consistent with the description of a set of waveguides in the PBG material as a bosonic reservoir, as done in Ref. [14].

The scope of this paper is to provide a microscopic derivation of the master equation when we deal with CQED involving PBG materials with cavity losses, in the framework of an appropriate non-Markovian theory. We will show that the dynamics of the system shows population trapping in the atomic excited state. This effect arises because the decay rates appearing in the microscopic dissipator are different.

The system we study consists of a two-level atom interacting with a cavity mode, where the cavity is coupled to a bosonic environment. The interaction between the atom and the system is described, at resonance and in units of $\hbar$, by the JC Hamiltonian $H_{J C}=$ $\left(\omega_{0} / 2\right) \sigma_{z}+\omega_{0} a^{\dagger} a+\Omega\left(a \sigma_{+}+a^{\dagger} \sigma_{-}\right)$, where $a^{\dagger}(a)$ is the creation (annihilation) operator of the mode, $\sigma_{-}=$ $|g\rangle\left\langle e\left|, \sigma_{+}=\right| e\right\rangle\langle g|$, and $\sigma_{z}=|e\rangle\langle e|-| g\rangle\langle g|$, while $|g\rangle$ and $|e\rangle$ denote the atomic ground and excited state respectively [15]. This model is valid as long as $\Omega \ll \omega_{0}$, so that one can neglect the counter-rotating terms $a \sigma_{-}$and $a^{\dagger} \sigma_{+}$. The cavity mode interacts with a bosonic reservoir, with Hamiltonian $H_{R}=\sum_{k} \omega_{k} b_{k}^{\dagger} b_{k}$, through the interaction Hamiltonian $H_{\text {int }}=\left(a+a^{\dagger}\right) \sum_{k} g_{k}\left(b_{k}+b_{k}^{\dagger}\right)$, which has the advantage of being treatable straightforwardly in the time-convolutionless formalism we are exploiting in this paper. Since the reservoir causing cavity losses is immersed in the PBG material, we expect its spectrum to be non-flat: to be rigorous, the master equation must be derived in the framework of a nonMarkovian theory. Using the second order of the timeconvolutionless (TCL) expansion [16, 17], and neglecting the atomic spontaneous emission and the Lamb shifts, the master equation, in the strong atom-cavity coupling regime, is equal to the Markovian one under the rotating wave approximation [18, 19], with the important difference that now the decay rates are time-dependent, indicating non-Markovian behavior. In this paper we focus on the case of one initial excitation and we consider 
a reservoir at zero temperature. In this case, the nonMarkovian master equation for the atom-cavity system density operator $\rho$ is:

$$
\begin{aligned}
\dot{\rho}(t) & =-i\left[H_{J C}, \rho\right] \\
& +\gamma\left(\omega_{0}+\Omega, t\right)\left(\frac{1}{2}\left|E_{0}\right\rangle\left\langle E_{1,+}|\rho(t)| E_{1,+}\right\rangle\left\langle E_{0}\right|\right. \\
& \left.-\frac{1}{4}\left\{\left|E_{1,+}\right\rangle\left\langle E_{1,+}\right|, \rho(t)\right\}\right) \\
& +\gamma\left(\omega_{0}-\Omega, t\right)\left(\frac{1}{2}\left|E_{0}\right\rangle\left\langle E_{1,-}|\rho(t)| E_{1,-}\right\rangle\left\langle E_{0}\right|\right. \\
& \left.-\frac{1}{4}\left\{\left|E_{1,-}\right\rangle\left\langle E_{1,-}\right|, \rho(t)\right\}\right),
\end{aligned}
$$

where $\left|E_{1, \pm}\right\rangle=(1 / \sqrt{2})(|1, g\rangle \pm|0, e\rangle)$ are the eigenstates of $H_{J C}$ with one total excitation, with energy $\omega_{0} / 2 \pm \Omega$, and $\left|E_{0}\right\rangle=|0, g\rangle$ is the ground state, with energy $-\omega_{0} / 2$. The time-dependent decay rates for $\left|E_{1,-}\right\rangle$ and $\left|E_{1,+}\right\rangle$ are $\gamma\left(\omega_{0}-\Omega, t\right)$ and $\gamma\left(\omega_{0}+\Omega, t\right)$ respectively.

If the system starts from the state $|0, e\rangle$, i.e., if the atom is initially excited and the cavity is initially empty, from Eq. (1) one can obtain the system density operator at all times:

$$
\begin{aligned}
& \rho(t)=\left(1-\frac{1}{2} \mathrm{e}^{-\frac{I_{-}(t)}{2}}-\frac{1}{2} \mathrm{e}^{-\frac{I_{+}(t)}{2}}\right)\left|E_{0}\right\rangle\left\langle E_{0}\right| \\
& +\frac{1}{2} \mathrm{e}^{-\frac{I_{-}(t)}{2}}\left|E_{1,-}\right\rangle\left\langle E_{1,-}\left|+\frac{1}{2} \mathrm{e}^{-\frac{I_{+}(t)}{2}}\right| E_{1,+}\right\rangle\left\langle E_{1,+}\right| \\
& -\frac{1}{2} \mathrm{e}^{-\frac{I_{-}(t)+I_{+}(t)}{4}}\left(\mathrm{e}^{2 i \Omega t}\left|E_{1,-}\right\rangle\left\langle E_{1,+}\right|+\text { h.c. }\right)
\end{aligned}
$$

where $I_{ \pm}(t)=\int_{0}^{t} \gamma\left(\omega_{0} \pm \Omega, t^{\prime}\right) d t^{\prime}$. From Eq. (2) it is possible to compute all the populations that we will show in the following. Below we will study the behavior of the non-Markovian time-dependent rates $\gamma\left(\omega_{0} \pm \Omega, t\right)$, which, through the quantities $I_{ \pm}(t)$, lead to population trapping.

As a model of environment at zero temperature with non-flat spectrum, we consider the Lorentzian distribution [16]:

$$
J(\omega)=\frac{1}{2 \pi} \frac{\alpha \lambda^{2}}{\left(\omega_{1}-\omega\right)^{2}+\lambda^{2}},
$$

where $\alpha$ is the system-environment coupling strength, and $\lambda$ is the width of the distribution, describing also the inverse of the reservoir memory time. The case of Lorentzian spectrum is analytically treatable, while capturing important features of the non-Markovian dynamics we are interested in, i.e. the time-dependence of the decay rates and their different stationary values. We consider the case in which the spectrum is peaked on the frequency of the state $\left|E_{1,-}\right\rangle$, i.e., $\omega_{1}=\omega_{0}-\Omega$, where $\omega_{0}$ is the atomic Bohr frequency and $\Omega$ is the Rabi splitting due to the JC interaction. The rate $\gamma(\omega, t)$ for a generic transition with Bohr frequency $\omega$ is equal to

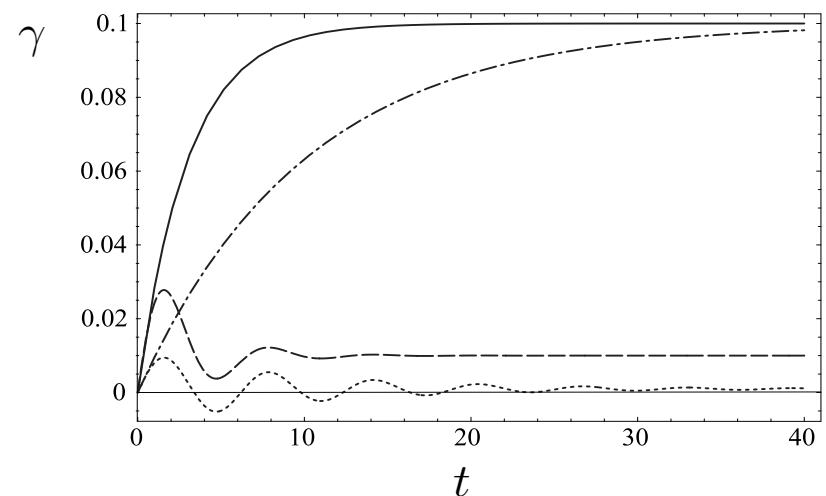

FIG. 1: Time-dependent decay rates (in units of $2 \Omega$ ) as a function of $t$ (in units of $(2 \Omega)^{-1}$ ) for the examples we are considering: ( $i) \lambda=2 \Omega / 3$, rates for $\left|E_{1,-}\right\rangle$ (solid line) and for $\left|E_{1,+}\right\rangle$ (dashed line); (ii) $\lambda=2 \Omega / \sqrt{99}$, rates for $\left|E_{1,-}\right\rangle$ (dashed-dotted line) and for $\left|E_{1,+}\right\rangle$ (dotted line).

$\gamma(\omega, t)=2 \operatorname{Re}\{\Gamma(\omega, t)\}$, where $\Gamma(\omega, t)$ is related to the spectral density $J(\omega)$ through the relation:

$$
\Gamma(\omega, t)=\int_{0}^{t} d \tau \int_{-\infty}^{+\infty} d \omega^{\prime} \mathrm{e}^{i\left(\omega-\omega^{\prime}\right) \tau} J\left(\omega^{\prime}\right) .
$$

By performing first the integral with respect to $\tau$ and then calculating the remaining integral by means of the method of the residues, we obtain the following expression for the decay rate $\gamma(\omega, t)$ :

$$
\begin{aligned}
& \gamma(\omega, t)=\frac{\alpha \lambda^{2}}{\left(\omega_{1}-\omega\right)^{2}+\lambda^{2}}\left\{1+\left[\frac{\omega_{1}-\omega}{\lambda} \sin \left(\omega_{1}-\omega\right) t\right.\right. \\
& \left.\left.-\cos \left(\omega_{1}-\omega\right) t\right] \mathrm{e}^{-\lambda t}\right\} .
\end{aligned}
$$

In particular, for $\omega_{1}=\omega_{0}-\Omega$ and substituting $\omega=$ $\omega_{0} \pm \Omega$, we obtain the decay rates for the two dressed states $\left|E_{1, \pm}\right\rangle$ :

$$
\gamma\left(\omega_{0}-\Omega, t\right)=\alpha\left(1-\mathrm{e}^{-\lambda t}\right)
$$

for $\left|E_{1,-}\right\rangle$ and

$$
\begin{aligned}
& \gamma\left(\omega_{0}+\Omega, t\right)=\frac{\alpha \lambda^{2}}{(2 \Omega)^{2}+\lambda^{2}}\left\{1+\left[\frac{2 \Omega}{\lambda} \sin 2 \Omega t\right.\right. \\
& \left.-\cos 2 \Omega t] \mathrm{e}^{-\lambda t}\right\}
\end{aligned}
$$

for $\left|E_{1,+}\right\rangle$.

From Eqs. (5)-(17) we clearly see the general behavior of the time-dependent rates: all the rates $\gamma(\omega, t)$ are zero at $t=0$, then they increase in time, till they reach stationary values for $t \gg \lambda^{-1}$. These stationary values are proportional to $J(\omega)$, i.e., they are equal to the rates one obtains from a Markovian theory. For these reasons, the quantity $\lambda^{-1}$ can be seen as the memory time of the 


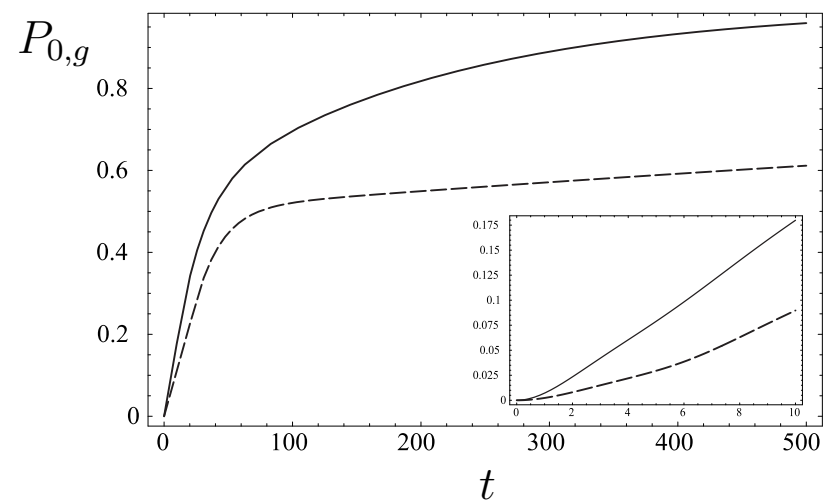

FIG. 2: Population of the ground state of the atom-cavity system, as a function of $t$ (in units of $(2 \Omega)^{-1}$ ) for the cases $\lambda=2 \Omega / 3$ (solid line) and $\lambda=2 \Omega / \sqrt{99}$ (dashed line). The inset shows the short-time dynamics.

system-reservoir interaction and non-Markovian effects are expected to occur for times shorter than $\lambda^{-1}$.

Figure 1 shows the decay rates for two cases, corresponding respectively to $\lambda /(2 \Omega)=1 / 3$ and $\lambda /(2 \Omega)=$ $1 / \sqrt{99}$, with $\alpha /(2 \Omega)=1 / 10$ for both cases. The condition on $\alpha$ assures the strong coupling regime, since the asymptotic decay rate of the state $\left|E_{1,-}\right\rangle$ is $\gamma\left(\omega_{0}-\right.$ $\Omega, \infty)=\alpha \ll 2 \Omega$ in both cases. As for the asymptotic decay rate of the state $\left|E_{1,+}\right\rangle$, in the first case $\lambda /(2 \Omega)=1 / 3$ corresponds to $\gamma\left(\omega_{0}+\Omega, \infty\right)=\gamma\left(\omega_{0}-\Omega, \infty\right) / 10$, while in the second case $\lambda /(2 \Omega)=1 / \sqrt{99}$ corresponds to $\gamma\left(\omega_{0}+\Omega, \infty\right)=\gamma\left(\omega_{0}-\Omega, \infty\right) / 100$. Note that in the case $\lambda /(2 \Omega)=1 / \sqrt{99}$ the decay rate for $\left|E_{1,+}\right\rangle$ also reaches negative values for short times: this is a typical feature of non-Markovian decay rates when the correlation time of the reservoir becomes large [16], and recently this has been connected to memory effects restoring coherence, for short times, within a quantum jump scheme [20].

By looking at the population $P_{0, g}$ of the system ground state $|0, g\rangle$, one can monitor the loss of energy from the atom-cavity system. Figure 2 shows the time evolution of $P_{0, g}$ for the two cases we are analyzing. Let us first consider the case $\lambda /(2 \Omega)=1 / 3$ (solid line). The longtime dynamics is well described by a decay law which is a sum of two exponentials, one with rate $\gamma\left(\omega_{0}-\Omega, \infty\right)$ and another one with rate $\gamma\left(\omega_{0}+\Omega, \infty\right)$, slower than the first one. When probing the population of the ground state, a proper analysis of the signal should allow to point out this feature and to distinguish it from the purely exponential decay predicted by the phenomenological dissipator for cavity losses in strong coupling. The inset in Fig. 2 shows the short-time evolution of the population under scrutiny, where the non-Markovian effects are evident. For very short times the rates start from zero and then increase linearly in time, as can be seen by expanding in power series of $t$ the rates in Eqs. (5)-(7). Hence the short-time behavior of the population is quadratic in time, in agreement with the predictions of the non-

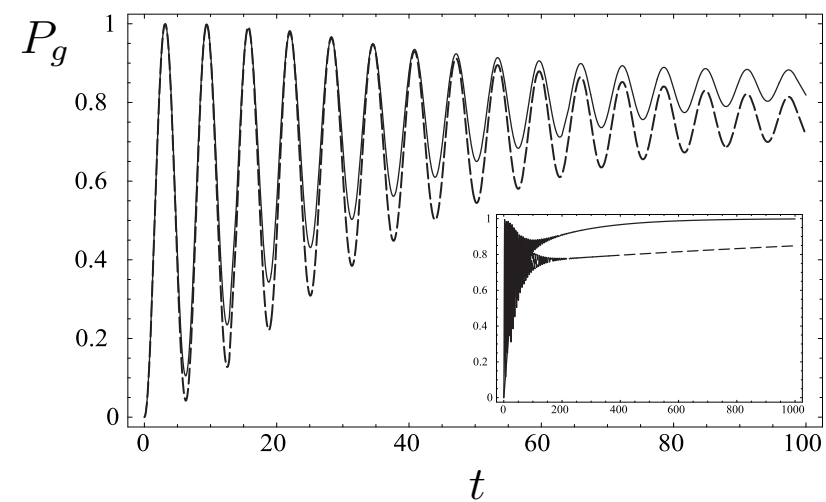

FIG. 3: Population of the atomic ground state, as a function of $t$ (in units of $(2 \Omega)^{-1}$ ), for the cases $\lambda=2 \Omega / 3$ (solid line) and $\lambda=2 \Omega / \sqrt{99}$ (dashed line). The inset shows the very long time behavior.

Markovian theory 16]. As time increases, signatures of the oscillations of the rate $\gamma\left(\omega_{0}+\Omega, t\right)$ may appear in the dynamics. For $\lambda /(2 \Omega)=1 / 3$ none of them is visible. For $\lambda / 2 \Omega=1 / \sqrt{99}$ (dashed line in Fig. 2) the main features of the dynamics remain unchanged but the short-time dynamics shows, along with the initial quadratic behavior, also slight signatures of the oscillations in the rate, consisting in a non-quadratic increase in the ground state population, with non-monotonic derivative. Such signatures are now visible both because the memory time of the reservoir is much longer than in the first case and because the decay rate of the state $\left|E_{1,+}\right\rangle$ also reaches negative values for some time intervals (see the dotted line in Fig. 1).

In the last case, the large difference between the two asymptotic decay rates leads to an interesting picture of the long-time dynamics. Indeed the ground state population first reaches the value of about $50 \%$ and stays close to this value for a very long time, then the system starts to decay again and the ground state population eventually goes to 1 (this restarting of the decay is not shown in Fig. (2). It is as if the second decay channel is turned on after a time of the order of $\gamma\left(\omega_{0}+\Omega, \infty\right)^{-1}$. Before this time the decay of $\left|E_{1,+}\right\rangle$ is almost completely inhibited, which is equivalent to the ideal situation wherein one of the two decay channels due to cavity losses is completely closed. This is at the origin of the population trapping due to cavity losses, as we are going to see.

Figure 3 shows the population of the atomic ground state $|g\rangle$. This is the quantity which is usually measured in standard CQED experiments after the atom has left the cavity [13]. As in standard CQED the population exhibits Rabi oscillations, but the dynamics of the decay of the oscillations is quite different. Since the two decay channels have different asymptotic rates, the state $\left|E_{1,-}\right\rangle$ decays before the state $\left|E_{1,+}\right\rangle$. Therefore there is a time interval, after the decay of $\left|E_{1,-}\right\rangle$ and before the complete decay of $\left|E_{1,+}\right\rangle$, wherein the Rabi oscillations are 
washed away by cavity losses, but some excitation is still present in the atom-cavity system. This is reflected in the fact that some population is trapped in the excited state of the atom for very long time, as shown in Fig. 3 . Comparing the cases $\lambda /(2 \Omega)=1 / 3$ and $\lambda / 2 \Omega=1 / \sqrt{99}$, we can see that in both cases the phenomenon of trapping is clearly visible. The difference is in the amount of trapping and in the length of the time interval wherein the trapping is present. The trapping time is longer in the second case, since the decay of $\left|E_{1,+}\right\rangle$ is much slower than in the first one. Anyway in both cases the trapping occurs for a time interval much longer than a Rabi period. The amount of trapping in the atomic excited state, in the first case, is a bit less than $20 \%$ while in the second case it is close to $25 \%$. It is clear that the amount of population trapping increases when the ratio between the two rates becomes smaller. The values we have shown in the latter case are very close to the limit values for population trapping due to cavity losses. Indeed the limiting case is the one wherein one rate is zero. In this case one of the two dressed states does not decay and the long-time population of the system ground state $|0, g\rangle$ is $50 \%$. The remaining population corresponds to the non-decaying dressed state, which is a superposition of the states $|0, e\rangle$ and $|1, g\rangle$ with equal weights. This means that the limit value achievable for the population trapped in the atomic excited state is equal to $25 \%$.

In conclusion, we have shown that a microscopic derivation of the non-Markovian master equation for the JC model when the cavity interacts with an external environment, allows one to predict population trapping due to cavity losses when the spectrum of the environment is not flat. It is important to note that the population trapping is a feature of the dynamics arising when the rates of the two dressed states are different. This means that even a non-Markovian extension of the phenomenological model used to describe cavity losses for the JC model, which would contain only one single time-dependent decay rate, could not predict this effect. Only a microscopic derivation of the master equation for the $\mathrm{JC}$ model does allow to correctly describe cavity losses for a non-flat spectrum of the environment and to predict the occurrence of population trapping in the dressed state, $\left|E_{1,+}\right\rangle$. This state is protected against cavity losses for a very long time, and its photon-atom entanglement could be exploited, e.g., to perform experiments on the violation of Bell's inequality. The long lifetime could indeed allow one to overcome the locality loophole, making it possible to send the atom away from the cavity far enough to be outside of the cavity lightcone [21]. A very important point is that our approach paves the way to the inclusion of cavity losses in the study of more complex forms of CQED in PBG materials. In particular our microscopic dissipator could be included in some schemes of quantum feedback control of the quantum dynamics of the atom-cavity system [22] and in the study of the physics of arrays of cavities. These latter systems have been recently exploited to simulate, through photon-atom dressed states, important models of condensed matter physics [23].

M.S. acknowledges financial support from CIMO for his stay in Turku during the period January-April 2007 and wishes to thank all the Quantum Optics Group members of the University of Turku for their kind hospitality. S.M., J.P. and K.-A.S. acknowledge financial support from the Academy of Finland (projects 108699, 115982, 115682), the Magnus Ehrnrooth Foundation and the Väisälä Foundation.
[1] H.I. Yoo and J.H. Eberly, Phys. Rep. 118, 239 (1985) and references therein; P.M. Radmore, Phys. Rev. A 26, 2252 (1982).

[2] Z. Deng and J.H. Eberly, Phys. Rev. A 34, 2492 (1986).

[3] J.I. Cirac and L.L. Sánchez-Soto, Phys. Rev. A 42, 2851 (1990); ibid. 44, 3317 (1991); G.S. Agarwal, Phys. Rev. Lett. 71, 1351 (1993).

[4] S. John, Phys. Rev. Lett. 58, 2486 (1987).

[5] D.G. Angelakis et al., Contemp. Phys. 45, 303 (2004).

[6] S. John and J. Wang, Phys. Rev. Lett. 64, 2418 (1990).

[7] S. John and T. Quang, Phys. Rev. A 50, 1764 (1994).

[8] T. Quang et al., Phys. Rev. Lett. 79, 5238 (1997).

[9] P. Lambropoulos et al., Rep. Prog. Phys. 63, 455 (2000).

[10] B. Lev et al., Nanotechnology 15, S556 (2004).

[11] L. Florescu et al., Phys. Rev. A 69, 013816 (2004).

[12] J.P. Reithmaier et al., Nature 432, 197 (2004); T. Yoshie et al., Nature 432, 200 (2004); D. Englund et al., Nature 450, 857 (2007); K. Srinivasan and O. Painter, Nature 450, 862 (2007).

[13] S. Haroche et al., in Fundamental Systems in Quantum Optics, ed. by J. Dalibard, J.-M. Raimond and J. ZinnJustin (North Holland, Amsterdam, 1992).
[14] S. Longhi, Phys. Rev. A 74, 063826 (2006).

[15] E.T. Jaynes and F.W. Cummings, Proc. IEEE 51, 89 (1963).

[16] H.-P. Breuer and F. Petruccione, The Theory of Open Quantum Systems (Oxford University Press, Oxford, 2002).

[17] The TCL formalism consists in a perturbative expansion, in the system-enivironment coupling constant, of an exact master equation for the open system under scrutiny. This approach is valid as long as such a coupling is weak enough, which in our case corresponds to the condition $\gamma\left(\omega_{0} \pm \Omega\right) \ll 2 \Omega[18]$.

[18] M. Scala et al., Phys. Rev. A 75, 013811 (2007).

[19] M. Scala et al., J. Phys. A: Math. Theor. 40, 14527 (2007).

[20] J. Piilo et al., arXiv:0706.4438v2.

[21] R. Pearle, Phys. Rev. D 2, 1418 (1970).

[22] J.E. Reiner et al., Phys. Rev. A 67, 042106 (2003); M.A. Armen and H. Mabuchi, Phys. Rev. A 73, 063801 (2006); D.A. Steck et al., Phys. Rev. A 74, 012322 (2006).

[23] M.J. Hartmann and M.B. Plenio, Nature Physics 2, 849 (2006); A.D. Greentree et al., Nature Physics 2, 
856 (2006); D.G. Angelakis et al., Phys. Rev. A 76, 031805(R) (2007); M.J. Hartmann and M.B. Plenio, Phys. Rev. Lett. 99, 103601 (2007); D. Rossini and R.
Fazio, Phys. Rev. Lett. 99, 186401 (2007). 